\title{
IMPLEMENTATION OF A SPECIFIC RATE CONTROLLER IN A FED-BATCH E. COLI FERMENTATION
}

\author{
I. Rocha ${ }^{1}$, A. C. A. Veloso ${ }^{1,2}$, S. Carneiro ${ }^{1}$, R. Costa $^{1}$ and E. C. Ferreira $^{1}$ \\ ${ }^{1} I B B$ - Institute for Biotechnology and Bioengineering, Centre of Biological Engineering, Universidade do \\ Minho \\ 4710-057 Braga, PORTUGAL \\ ${ }^{2}$ Escola Superior Agrária de Bragança, Campus de Santa Apolónia, Apartado 1172 \\ 5301-854 Bragança, PORTUGAL \\ e-mail: \{irocha, anaveloso, soniacarneiro, rafacosta, ecferreira\}@deb.uminho.pt
}

\begin{abstract}
The specific growth rate is one of the most important process variables characterizing the state of microorganisms during fermentations mainly because the biosynthesis of many products of interest is often related with the values assumed by this parameter. In the particular case of the fed-batch operation of Escherichia coli for the production of recombinant proteins, it is important to maintain the specific growth rate below a certain threshold in order to avoid the accumulation of acetic acid throughout the fermentation and, additionally, it is often argued that both pre- and the post-induction specific growth rates should be closely controlled in order to achieve maximum productivities of the desired recombinant protein.

In a previous work the authors have developed and validated by simulations a strategy for the automatic control of the specific growth rate in $E$. coli fed-batch fermentations based on an asymptotic observer for biomass and on developed estimators for the specific growth rates. The main purpose of the present work was to implement experimentally the developed observer, estimator and controller in a real fed-batch fermentation process. For that purpose a data acquisition and control program was developed in LabVIEW that allows the acquisition of the necessary on line data (off gas and dissolved oxygen concentration and culture weight) and the calculation of the feeding rates using the developed equations. The feedforward-feedback controller developed was able to keep the culture growing in an exponential phase throughout the fermentation without accumulation of glucose and acetate. Copyright 2008 IFAC
\end{abstract}

Key-words: Soft-sensors, Specific growth rate estimation, Non-linear systems, Fed-batch fermentation, E. coli, feedforward-feedback controller.

\section{INTRODUCTION}

The specific growth rate is one of the most important process variables characterizing the state of microorganisms during fermentations mainly because the biosynthesis of many products of interest is often related with the values assumed by this variable. As opposed to batch fermentation, in fed-batch cultures it is possible to manipulate the specific growth rate at an appropriate value providing a desirable metabolic condition, resulting in maximum productivity.

Additionally, for certain types of measurements, of vital importance in the post-genomic era (Bro and Nielsen, 2004), like mRNA abundance or the analysis of the fluxome and proteome, microbial cultures have to be sampled at a pseudo steady-state condition that can be obtained by imposing a fixed 
specific growth rate, either in continuous or fed-batch cultures. The advantage of fed-batch cultures is that they allow much higher biomass concentrations, being therefore much closer to the industrial processes.

In the particular case of the fed-batch operation of Escherichia coli for the production of recombinant proteins, it is important to maintain the specific growth rate below a certain threshold in order to avoid the accumulation of acetic acid throughout the fermentation (Jana and Deb, 2005) and, additionally, it is often argued that both pre- and the postinduction specific growth rates should be closely controlled in order to achieve maximum productivities of the desired recombinant protein (Curless et al., 1990; Sanden et al, 2003; Lim and Jung, 1998).

In order to keep the specific growth rate at a predetermined value, the most common approach is to apply a feed-forward exponential feeding strategy, where the nutrients required by the culture for achieving the desired growth rate are pre-determined and satisfied at any moment (Martinez at al., 1998, Yang, 1992). However, this feed-forward method has several limitations mainly related with the likely occurrence of external perturbations or variations on culture parameters.

Therefore, the development of reliable algorithms for the feedback automatic control of the specific growth rate in fed-batch systems is of paramount importance in fermentation technology. However, the performance of such algorithms is critically dependent on a reliable determination of the specific growth rate, which cannot be obtained directly from common fermentation measurements mainly due to the lack of reliable sensors for the determination of biomass concentration. A combination of a reliable model of the process and on-line data is therefore often necessary for the estimation of both biomass concentration and specific growth rate.

Some algorithms used for on-line estimation of reaction rates using biomass concentration or other correlated variables measurements have been proposed. Pomerleau and Perrier (1990) and Pomerleau and Perrier (1992) proposed and validated experimentally an on-line estimation algorithm for multiple reaction rates. This procedure was applied to baker's yeast fermentation, and the algorithm required the on-line measurement of two or three state variables. Also, in Ljubenova (1999) the author describes a methodology for the design of a new parameter estimator of biomass growth rate and yield coefficient for oxygen consumption on the basis of the theory of adaptive estimation, for a class of aerobic bioprocesses in fed-batch or continuous mode. In Ljubenova and Ignatova (1994) and
Lubenova et al (2003), the authors proposed an approach for on-line growth rate estimation for a class of aerobic batch processes with dissolved oxygen control in the culture medium. The only required on-line measurement is the oxygen consumption rate. An adaptive model-based algorithm for the on-line estimation of reaction rates is described by Oliveira et al. (2002), considering the yield coefficients invariable and known, based on the approach of Bastin and Dochain (1990) to stirred tank reactors.

Regarding the development of control algorithms for the specific growth rate, there are very few examples where the closed loop control has been applied to $E$. coli or to other relatively complex bioprocesses. In Levisauskas (2001) and Levisauskas et al. (1996) the authors have developed a strategy for automatic control of the specific growth rate in fed-batch cultivation that requires the on-line monitoring of OUR or CER and feed rate and volume of the fermentation broth. This strategy was applied to fedbatch fermentation of $E$. coli.

In a previous work (Rocha et al, 2006 and Rocha and Ferreira, 2006) we have developed and validated by simulations a strategy for the automatic control of the specific growth rate in $E$. coli fed-batch fermentations based on an asymptotic observer for biomass and on developed estimators for the specific growth rates.

The main purpose of the present work was to implement experimentally the developed observer, estimator and controller in a real fed-batch fermentation process. For that purpose a data acquisition and control program was developed in LabVIEW that allows the acquisition of the necessary on line data (exhausted and dissolved oxygen concentration and culture weight) and the calculation of the feeding rates using the developed equations.

\section{PROCESS MODELLING}

The development of the mathematical model for the fed-batch fermentation of recombinant $E$. coli was based on the assumption that the aerobic growth of the microorganism can follow three main different metabolic pathways (Rocha and Ferreira, 2002): oxidative growth on glucose, fermentative growth on glucose, and oxidative growth on acetate, the corresponding dynamical model for fed-batch fermentation being represented as follows: 


$$
\frac{d}{d t}\left[\begin{array}{c}
X \\
S \\
A \\
O \\
C
\end{array}\right]=\left[\begin{array}{ccc}
1 & 1 & 1 \\
-y_{1} & -y_{2} & 0 \\
0 & y_{3} & -y_{4} \\
-y_{5} & -y_{6} & -y_{7} \\
y_{8} & y_{9} & y_{10}
\end{array}\right]\left[\begin{array}{l}
\mu_{1} \\
\mu_{2} \\
\mu_{3}
\end{array}\right] X-D\left[\begin{array}{c}
X \\
S \\
A \\
O \\
C
\end{array}\right]+\left[\begin{array}{c}
0 \\
\frac{F_{\text {in }}}{W} S_{\text {in }} \\
0 \\
O T R \\
-C T R
\end{array}\right]
$$

where $\mu_{1}, \mu_{2}$, and $\mu_{3}$ are the specific growth rates; $y_{i}$ are the yield (stoichiometric) coefficients; $F_{\text {in }}$ and $S_{i n}$ are the substrate feed rate and the influent glucose concentration, respectively; $W$ is the culture medium weight. CTR is the carbon dioxide transfer rate from liquid to gas phase, and $O T R$ is the oxygen transfer rate from gas to liquid phase.

The variation of the culture medium weight with the time is given by:

$$
\frac{d W}{d t}=F
$$

Where $F$ includes weight variations due to the substrate feed rate, the amount of culture removed or added during sampling, base and acid additions, evaporation and mass taken from the reactor due to gas exchanges, that cannot be considered negligible in small-scale high-cell density reactors.

A full description of the kinetic model used for the fed-batch growth of E. coli can be found in (Rocha, 2003).

As a consequence of the characteristics of the oxidative bottleneck, the three metabolic pathways represented in the mathematical model do not occur simultaneously in the cell, resulting in four partial models corresponding to different metabolic regimens:

- Simultaneous oxidative and fermentative growth on glucose $\left(\mu_{1}, \mu_{2}>0\right)$

- Oxidative growth on glucose $\left(\mu_{1}>0\right)$

- Oxidative growth on acetate and glucose simultaneously $\left(\mu_{1}, \mu_{3}>0\right)$

- Oxidative growth on acetate $\left(\mu_{3}>0\right)$

\section{BIOMASS OBSERVER AND SPECIFIC GROWTH RATE ESTIMATORS}

Due to the inexistence of reliable sensors for the determination of biomass concentration in $E$. coli fermentations, the estimation of the specific growth rate necessarily begins with the observation of that state variable from available on-line data. Although in the authors' lab there exists the possibility of measuring on-line all the remaining state variables from equation 1 (Rocha and Ferreira, 2002), the more reliable and frequent measurements are related with exhaust gases, i.e., CTR and OTR, apart from culture weight. Additionally, both the biomass observer and the specific growth rate estimators assume that the yield coefficient matrix $Y$ is known.

Therefore, using the information available on-line and according to Veloso et al. (2005) it is possible to obtain biomass estimation using either Extended Kalman Observers (EKO) or Asymptotic Observers (AO) only if a partial model is considered. Taking into account that acetate consumption is very often negligible in these processes and that the specific growth rate is usually intended to be kept below its critical value (the threshold above which acetate is produced), the "oxidative growth on glucose" regimen is the only one considered. Additionally, and although the performance of the EKO was shown to be slightly superior for this process, the AO was selected for biomass estimation, as it requires no knowledge on the kinetic structure of the model and also due to the fact that no tuning parameters are required.

The AO allows reconstructing the missing state variables even when the process is not exponentially observable and the kinetics are unknown (Bastin and Dochain, 1990). Biomass estimation requires the integration of one differential equation associated with the auxiliary variable $Z_{l}$ where its dynamics is independent of growth rate vector $r(\xi, t)$ :

$$
\frac{d \hat{Z}_{1}}{d t}=-D \hat{Z}_{1}+\theta_{1} O T R
$$

The variable $\theta_{l}$ is function of the yield coefficients matrix as follows:

$$
\theta_{1}=\frac{-1}{y_{5}}
$$

The biomass estimate is then given by:

$$
\hat{X}=\hat{Z}_{1}+\theta_{1} O
$$

The design of specific growth rate estimators is based on the formulation proposed by Bastin and Dochain (1990) reformulated by Pomerleau and Perrier (1990) so that a decoupling of the dynamic model (equation 1) from the growth rate is achieved. The estimation of the specific growth rate corresponding to equation 1 will then come:

$$
\begin{aligned}
\frac{d \hat{\psi}_{1}}{d t} & =\hat{\mu}_{1} \hat{X}-D \hat{\psi}_{1}+\theta_{1} O T R+\omega_{1}\left(\psi_{1}-\hat{\psi}_{1}\right) \\
\frac{d \hat{\mu}_{1}}{d t} & =\omega_{2}\left(\psi_{1}-\hat{\psi}_{1}\right)
\end{aligned}
$$

with:

$$
\psi_{1}=\theta_{1} O
$$


The calculation of the gains $\omega_{\mathrm{i}}$ for each time instant $i$ is made such that a second order dynamics (Oliveira et al., 1996) is obtained:

$$
\begin{aligned}
& \omega_{1, i}=2 \zeta_{1} / \tau_{1}-\frac{X_{i}-X_{i-1}}{T X_{i}} \\
& \omega_{2, i}=\left(X_{i} \tau_{1}^{2}\right)^{-1}
\end{aligned}
$$

where $T$ is the integration step. Therefore, the implementation of these algorithms requires the tuning of 2 parameters $\left(\zeta_{1}\right.$ and $\left.\tau_{1}\right)$.

\section{CONTROL ALGORITHM}

Due to the growth of the microorganisms, timevarying characteristics of the cultivation process occur and have to be taken into consideration if a controller is to be implemented. Using a feedforward-feedback controller these time-varying characteristics have to be kept in view in the feedforward contribution.

The deduction of the feed-forward component of the control equation is obtained from the mass balance for the substrate $S$ of equation 1, when only the oxidative growth on glucose pathway is active considering that along the fed-batch phase $S=0$ and $d S / d t=0$. This exponential feeding strategy is corrected by incorporating an appropriate feedback control action (in this case a proportional action), resulting in a control equation very similar to the optimal adaptive control approach described by Smets et al. (2004):

$$
F_{i n}=\frac{y_{1} \mu_{s e t} \hat{X}(t) W(t)}{S_{i n}}-\tau_{\mu} \frac{\hat{\mu}(t)-\mu_{s e t}}{S_{i n}} W(t)
$$

where $\mu_{\text {set }}$ is the desired growth rate that has necessarily to be smaller than the maximum value of $\mu_{1}$.

For the application of this control law, $y_{1}$ and $S_{\text {in }}$ are considered known and invariant, while $W(t)$ is obtained on-line using a balance under the bioreactor. Estimates of $X(t)$ and $\mu(t)$ are obtained using equations 5 and 6 , respectively.

\section{IMPLEMENTATION OF THE ESTIMATION AND CONTROL ALGORITHMS IN LABVIEW}

\subsection{Experimental Conditions and Equipment}

The bioreactor is a Biostat MD fermenter from $B$. Braun Biotech (Germany) with 5 L of capacity connected to a Digital Control Unit. The E. coli fermentation is operated in fed-batch mode, with an initial weight of $3 \mathrm{~kg}$ and the addition of $2 \mathrm{~kg}$ of feeding solution. A modified M9 medium with addition of trace metals and vitamins is used for the batch cultivation of the microorganism and a solution of glucose $(250 \mathrm{~g} / \mathrm{kg})$ and ammonia is added during the fed-batch phase. The set-points for the environmental properties are $37^{\circ} \mathrm{C}$ for temperature, $\mathrm{pH} \mathrm{7,} \mathrm{and} 30 \%$ of saturation for dissolved oxygen. For off gas measurements, the BCP sensors for $\mathrm{O}_{2}$ and $\mathrm{CO}_{2}$ from Bluesens, Germany are used. Two balances are used for the measurement of the total weight of the fermenter and for weighting the feeding solution reservoir.

\subsection{Hardware and Software}

LabVIEW programs consist of three parts: the front panel, which is the interface between the program and the user; the block diagram (the program itself) and the icon connector, which is responsible for data flow between routines. Together, these three elements integrate a Virtual Instrument (VI), the basic element of a LabVIEW program. Several VIs were constructed to acquire data from each specific instrument, and then called from a superior hierarchic level in the supervisor program. All the control routines were also implemented in this main program.

RS-232 protocol is used for the communication with the fermenter's DCU. During a fermentation, dissolved oxygen concentration, temperature, $\mathrm{pH}$, stirrer speed and air flow rate values are converted into ASCII strings and received by the corresponding VI, which continuously decodes the information, stores it and leaves it ready for being used for control purposes. RS-232 and string interpretation are also used in the communication with the balances for the acquisition of the weight measurements.

Information from Bluesens equipment is acquired by an analog-to-digital converter board from National Instruments, USA (PCI 6024E). A VI was built that integrates this information with data from the fermenter's weight for the on-line calculation of both CTR and OTR. Each OTR and CTR value is a result of 12 raw data points that are filtered for outliers and averaged.

The variables obtained from the different sources are integrated in the supervisory VI (Fig 1) as input information for the estimation and control algorithms, being the controller output the set-point for the feeding pump. Each iteration takes 1 minute and therefore the setpoint for the feeding rate can change at that rate. The calculations of the estimated biomass concentration and specific growth rate in equations 5 and 6 and of the control law given by equation 9 are performed in a $\mathrm{C}$ script embedded in 
the supervisor LabVIEW VI, using a recursive calculation method, where the values of the relevant variables are stored for the next iteration. Both initial values of estimated variables and tuning parameters were obtained previously in simulation studies (Rocha et al, 2006 and Rocha and Ferreira, 2006).

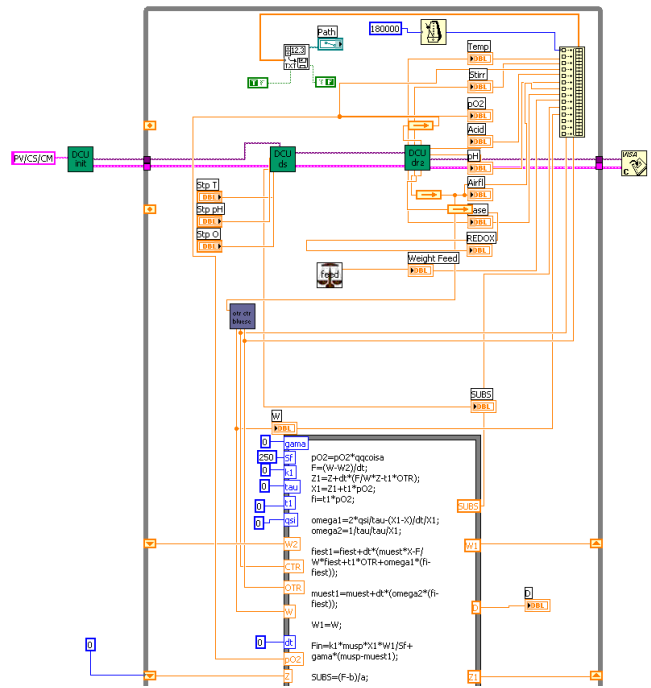

Figure 1 Block diagram of the supervisor VI showing the subVI's used to communicate with the relevant equipment and the embedded $\mathrm{C}$ script for the implementation of the biomass observer, the specific growth rate estimator and the control law.

\section{RESULTS AND DISCUSSION}

The setup described was used to run a fed-batch fermentation where the setpoint for the specific growth rate was fixed at $0.11 \mathrm{~h}^{-1}$, a value that is below the critical specific growth rate for $E$. coli. It can be seen from Fig. 2 that the asymptotic observer for biomass was able to accurately predict biomass concentration until the eighth hour of feeding, slightly over predicting that variable from that point on.

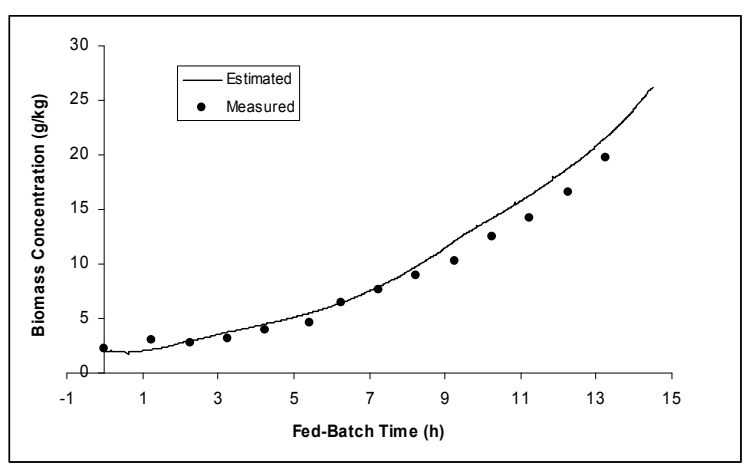

Figure 2 Performance of the on-line observer for biomass concentration during a controlled fed-batch fermentation.

It is also clear from figs 2 to 4 that the specific growth rate controller was able to maintain the cells growing in an exponential phase during the feeding period. Also, both glucose and acetate concentrations were always kept very close to zero. While glucose amounts were always under detectable levels (below $0.1 \mathrm{~g} / \mathrm{kg}$ ), acetate reached a maximum of $0.13 \mathrm{~g} / \mathrm{kg}$, meaning that the specific growth rate was kept below its critical limit by the controller.

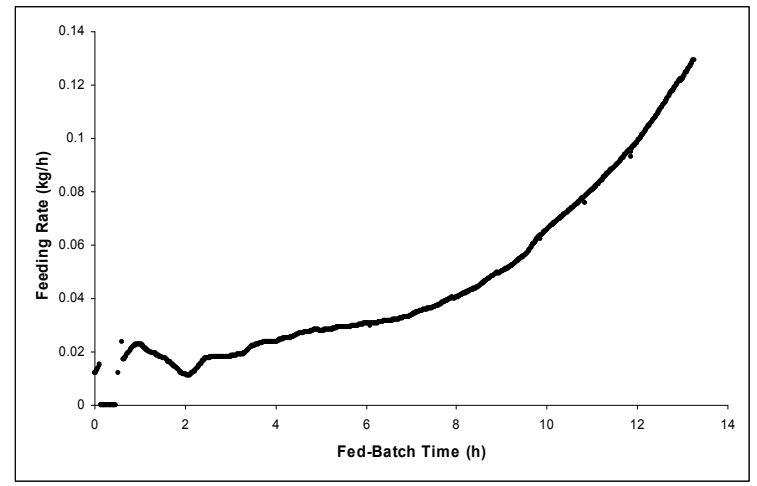

Figure 3 Feeding rate profile obtained by the application of the feedforward-feedback controller for the specific growth rate.

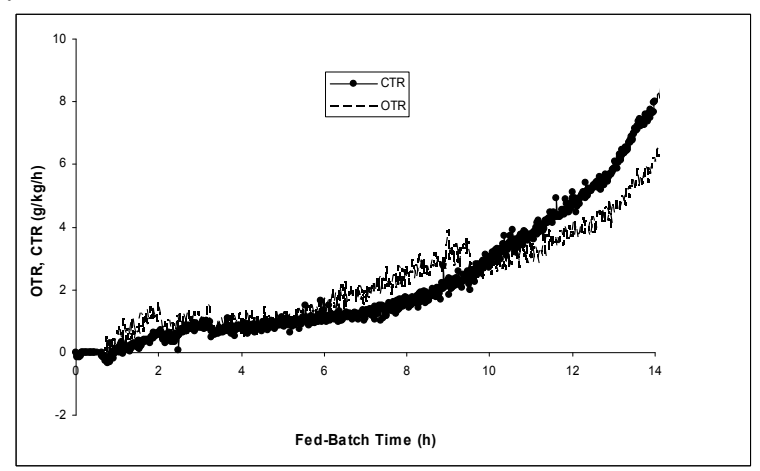

Figure 4 Oxygen and carbon dioxide transfer rates measured online and used for the observation of biomass and estimation of the specific growth rate.

\section{CONCLUSIONS AND FUTURE WORK}

With the developed algorithms, it was possible to perform a controlled fed-batch fermentation process of $E$. coli using only on-line data from dissolved oxygen, OTR and culture weights. The feedforwardfeedback controller was able to keep the culture growing in an exponential phase throughout the fermentation without accumulation of glucose and acetate.

Future work involves the evaluation of the robustness of the methodology developed when the setpoint is changed and also the implementation of the controller considering also the "oxidative and fermentative growth on glucose" regimen. 


\section{ACKNOWLEDGMENTS}

This work was supported by FCT - Portuguese Science Foundation under the scope of the recSysBio Project (POCI/BIO/60139/2004).

\section{REFERENCES}

Bastin, G. and Dochain, D. On-line estimation and adaptive control of bioreactors. Elsevier Science Publishers, Amsterdam (1990).

Bro, C. and Nielsen, J. Impact of 'ome' analyses on inverse metabolic engineering. Metabolic Engineering 6, 204-211 (2004).

Curless, C., Pope, J. and Tsai, L. Effect of preinduction specific growth rate on recombinant alpha consensus interferon synthesis in Escherichia coli. Biotechnology Progress 6, 149-152 (1990).

Jana, S. and Deb, J. K. Strategies for efficient production of heterologous proteins in Escherichia coli. Applied Microbiology and Biotechnology 67, 289-298 (2005).

Levisauskas, D. Inferential control of the specific growth rate in fed-batch cultivation processes. Biotechnology Letters 23, 1189-1195 (2001).

Levisauskas, D., Simutis, R., Borvitz, D. and Lübert, A. Automatic control of the specific growth rate in fed-batch cultivation processes based on an exhaust gas analysis. Bioprocess Engineering 15, 145-150 (1996).

Lim, H.-K. and Jung, K.-H. Improvement of heterologous protein productivity by controlling postinduction specific growth rate in recombinant Escherichia coli under control of the PL promoter. Biotechnology Progress 14, 548-553 (1998).

Ljubenova, V. and Ignatova, M. An approach for parameter estimation of biotechnological processes. Bioprocess Engineering 11, 107-113 (1994).

Lubenova, V. N. Stable adaptive algorithm for simultaneous estimation of time-varying parameters and state variables in aerobic bioprocesses. Bioprocess Engineering 21, 219-226 (1999).

Lubenova, V., Rocha, I. and Ferreira, E. C. Estimation of multiple biomass growth rates and biomass concentration in a class of bioprocesses. Bioprocess and Biosystems Engineering 25, 395-406 (2003).

Martinez, A., Ramirez, O. and Valle, F. Effect of growth rate on the production of B-galactosidase from Escherichia coli in Bacillus subtilis using glucose-limited exponentially fedbatch cultures. Enzyme and Microbial Technology 22, 520-526. 1998.
Oliveira, R., Ferreira, E. C., Feyo de Azevedo, S. Stability, dynamics of convergence and tuning of observer-based kinetics estimators. Journal of Process Control 12:2, (2002), 311-323.

Oliveira, R., Ferreira, E.C., Oliveira, F, Feyo de Azevedo, S. A Study on the Convergence of Observer-Based Kinetic Estimators in Fed-Batch Fermentations. Journal of Process Control, 6:6, (1996), 367-371.

Pomerleau, Y. and Perrier, M. Estimation of multiple specific growth rates in bioprocesses. AIChE Journal 36, 207-215 (1990).

Pomerleau, Y. and Perrier, M. Estimation of multiple specific growth rates: experimental validation. AIChE Journal 38, (1992), 1751-1760.

Rocha, I. Model-based strategies for computer-aided operation of recombinant E. coli fermentation. 2003. Universidade do Minho, $\mathrm{PhD}$ Thesis.

Rocha, I. and Ferreira, E. C. On-line simultaneous monitoring of glucose and acetate with FIA during high cell density fermentation of recombinant $E$. coli. Analytica Chimica Acta 462, (2002), 293-304.

Rocha, I., Veloso, A.C.A., Ferreira, E.C. Design of estimators for specific growth rate control in a fedbatch E. coli fermentation. Proceedings 5th MATHMOD Vienna - 5th Vienna Symposium on Mathematical Modelling, Austria, February 8-10, 2006.

Rocha, I., Ferreira, E.C. Specific growth rate regulation in a simulated fed-batch E. coli fermentation. CONTROLO2006 - 7th Portuguese Conference on Automatic Control, Lisboa, 11-13 de Setembro, 6 pp., 2006.

Sanden, A. M. et al. Limiting factors in Escherichia coli fed-batch production of recombinant proteins. Biotechnology and Bioengineering 81, 158-166 (2003).

Smets, I. Y., Claes, J. E., November, E. J., Bastin, G. P. and Van Impe, J. F. Optimal adaptive control of (bio)chemical reactors: past, present and future. Journal of Process Control 14, 795-805 (2004).

Veloso, A. C. A., Rocha, I. and Ferreira, E. C. Design of on-line state estimators for a recombinant E. coli fed-batch fermentation. Horacek, P., Simandl, M, and Zitek, P. Preprints of the 16th IFAC World Congress. 2005. Prague - Czech Republic.

Yang, X.-M. Optimization of a cultivation process for recombinant protein production by Escherichia coli. Journal of Biotechnology 23, 271-289 (1992). 